\title{
Synthesis of oligodeoxynucleotides bearing structurally defined adducts of mutagens
}

\author{
Thomas M. Harris and Constance M. Harris
}

Department of Chemistry and Center in Molecular Toxicology, Vanderbilt University, Nashville, TN 37235

E-mail: harristm@toxicology.mc.vanderbilt.edu

\section{Dedicated to Professor Rudolph A. Abramovitch on the occasion of his $70^{\text {th }}$ birthday (received 08 May 01; accepted 07 Aug 01; published on the web 15 Aug 01)}

\begin{abstract}
Methodology is reviewed for the preparation of oligodeoxynucleotides bearing site-specific adducts of mutagens. Examples are presented of the use of the three general synthetic approaches: (1) direct adduction of oligonucleotides, (2) introduction of adducted nucleosides during oligonucleotide assembly, and (3) post-oligomerization methods in which an oligonucleotide is assembled containing an electrophilic equivalent of the target nucleoside, followed by formation of the adduct by condensation with a nucleophilic equivalent of the mutagen. The merits and limitations of the three methods are discussed.
\end{abstract}

Keywords: Oligodeoxynucleotides, adducted nucleosides, adduction

\section{Table of Contents}

1. Introduction

2. Direct Adduction

3. Adducted nucleosides

4. Post-oligomerization

5. Conclusions

\section{Introduction}

Mutagenic compounds act on DNA most commonly by forming adducts; other types of damage include oxidation, deamination and depurination of the nucleosides. For the systematic study of the biochemical and biological effects of these various types of DNA damage, DNA is needed in which the adducts or other damage is placed in the DNA in a structurally specific manner. The structural modification of the DNA must be specific with regard to a) sequence, b) site of reaction on the nucleoside and on the mutagen, and c) the configuration of stereogenic centers. 
The past decade has seen intense effort to develop convenient and versatile synthetic approaches to oligodeoxynucleotides containing structurally defined adducts. The adducted oligonucleotides have been useful not only for establishing the biological effects of the various lesions, but also for biophysical studies, in particular two-dimensional NMR, with the goal of establishing the structural basis for replication and other types of biological processing. This article reviews the various synthetic approaches that can be taken with particular focus on the methods developed in the laboratory of the authors.

Three general strategies have arisen for preparation of DNA bearing structurally defined adducts. One is direct adduction of oligodeoxynucleotides by the activated mutagen. A second involves preparation of a structurally defined adducted deoxynucleoside, use of it for preparation of the phosphoramidite reagent and then assembly of the oligodeoxynucleotide. A third approach involves assembly of the oligonucleotide containing a unique, reactive nucleoside, which is then used in post-oligomerization reactions for in situ assembly of the adduct. Each of these methods has unique strengths and weaknesses, which are pointed out in the examples discussed below.

\section{Direct Adduction}

Without doubt, the simplest way to prepare adducted oligodeoxynucleotides is direct reaction with an activated mutagen. However, there are severe limitations on the generality of this method. A key limitation is the need for a high level of chemoselectivity. The electrophile must react specifically with a single type of nucleoside and only at a single site in the nucleoside, for example only with deoxyguanosine at $\mathrm{N} 7$ or only at $\mathrm{N}^{2}$. Furthermore, the electrophile must react in a single way. For example an unsymmetrical epoxide needs to react at only one end and with fixed stereochemistry of attack. In reality, few electrophiles fully meet this goal but some come close enough for the method to be useful. Even under the best of circumstances, a further limitation is that the oligonucleotide can contain no more than one, two or at most three target nucleosides.

Aflatoxin $\mathrm{B}_{1}$ presents a near perfect situation. It is converted to the active form, the exo-8,9epoxide, by cytochrome $\mathrm{P} 450$ or in the laboratory by dimethyldioxirane. ${ }^{1}$ The epoxide reacts with nucleophiles exclusively by attack at $\mathrm{C} 8$ with inversion of configuration. The epoxide reacts rapidly with water, $\mathrm{t}_{1 / 2}=<1 \mathrm{sec}$ at ambient temperature and $\mathrm{pH} \mathrm{7.0,}{ }^{2}$ but reaction with duplex DNA occurs much more rapidly leading to high adduction yields. ${ }^{1,3}$ The reaction occurs almost exclusively at the N7 position of guanine. Excellent results have been obtained in preparation of adducted oligonucleotides in cases where only one guanine is present in a chain but the presence of multiple guanines creates separation problems since all can potentially react with the epoxide (Scheme 1). Two solutions have been found for this problem. One involves use of a triple helix strategy to protect the N7 positions of some of the guanines while others are permitted to react. ${ }^{4}$ 

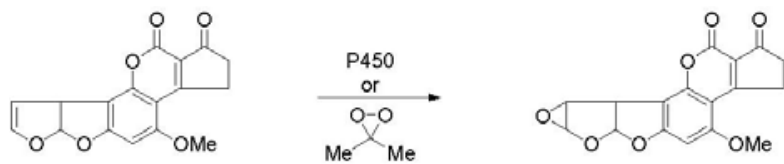

$5^{5}$-d(ATCGAT) $)_{2}$

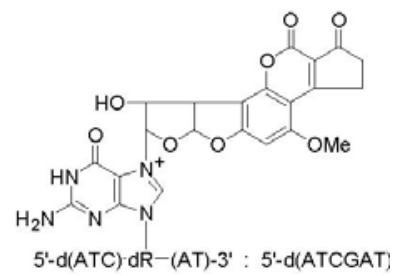

Scheme 1. Direct adduction procedure for preparation of an oligodeoxynucleotide containing a guanine $\mathrm{N} 7$ adduct of the epoxide of aflatoxin $\mathrm{B}_{1}$.

A second capitalizes on the fact that the transition state for adduction involves intercalation of the aflatoxin epoxide on the 5 ' face of the target guanine. ${ }^{5}$ The strategy employs intercalators appended to the complementary strand such that they block entry of the aflatoxin epoxide adjacent to those guanines where adduction is not wanted leaving the remaining ones capable of reaction. ${ }^{6}$ The success of both methods is dependent on the highly structured transition state for the aflatoxin adduction reaction and is not likely to be widely applicable with other electrophiles. It should be mentioned in passing that the adduct of aflatoxin epoxide is unstable to acid, base and heat. These limitations on reaction conditions make direct adduction the method of choice for assembly of adducted oligonucleotides.

Another example where direct adduction has been successful is the reactions of the diol epoxides of certain of the polycyclic aromatic hydrocarbons. The chemoselectivity of the diol epoxides varies with the structure of the hydrocarbon and configurations of the epoxide and hydroxy groups. The diol epoxides react exclusively via the benzylic carbon but yield varying mixtures of retention and inversion of configuration in the adduct and varying ratios of adduction at the $\mathrm{N}^{2}$ position of guanine and $\mathrm{N}^{6}$ position of adenine, depending on the structure of the PAH diol epoxide. Excellent results have been obtained with the dihydrodiol epoxides of benzo $[a]$ pyrene when the reactions are carried out on single stranded oligodeoxynucleotides which give high selectivity for the trans adduct at the $\mathrm{N}^{2}$ position of guanine (Scheme 2). ${ }^{7}$ Direct adduction has also been used to prepare oligonucleotides containing adducts of a diol epoxide of benzo $[c]$ phenanthrene which shows preference for reaction at $\mathrm{N}^{6}$ of deoxyadenosine. ${ }^{8}$
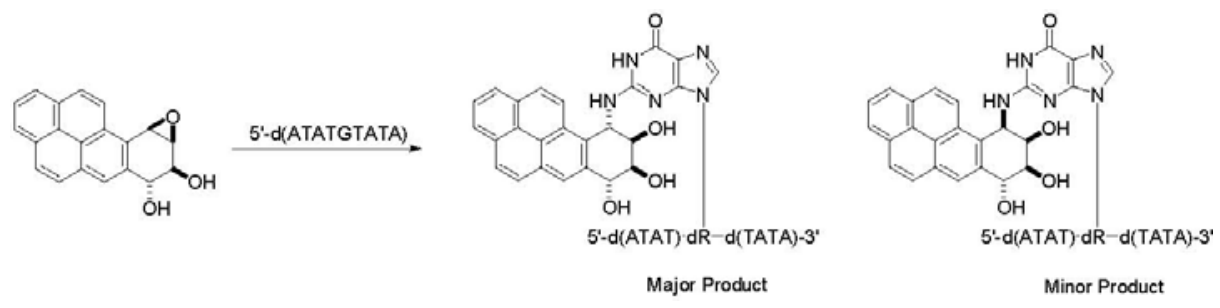

Scheme 2. Direct adduction procedure for preparation of an oligodeoxynucleotide containing the 
$\mathrm{N}^{2}$ guanine adducts of the $(+)$-anti dihydrodiol epoxide of benzo[a]pyrene.

A third example of direct adduct involves the formation of the guanine $\mathrm{C} 8$ adduct of $\mathrm{N}$ acetyl-2-aminofluorene. An oligodeoxynucleotide containing three guanine residues has been treated with $\mathrm{N}$-acetoxy-N-acetyl-2-aminofluorene (Scheme 3). The three monoadducts were found to be separable by HPLC. Three bis adducts and one tris were also formed. While the separability of sequence isomers is in no way assured, the success in this case provides hope that favorable results may be obtained using other electrophiles. ${ }^{9}$

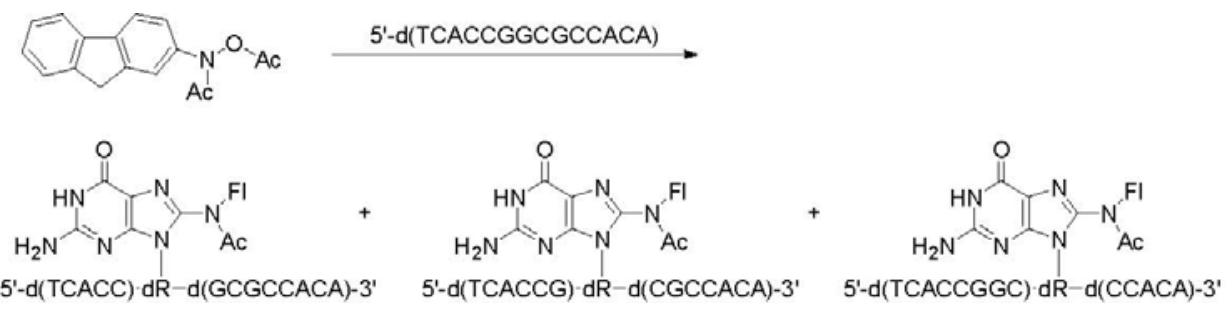

Scheme 3. Direct adduction procedure for preparation of an oligodeoxynucleotide containing guanine $\mathrm{C} 8$ adducts of $\mathrm{N}$-acetyl-2-aminofluorene ( $\mathrm{Fl}=2$-fluorenyl).

\section{Adducted nucleosides}

The second major strategy for preparation of adducted oligonucleotides involves preparation of adducted nucleosides and then using them to assemble oligonucleotides. Enzymatic methods can be used to introduce the adducted nucleoside, ${ }^{10}$ but most commonly chemical methods are used to assemble the oligonucleotide. In favorable cases the chemical approach is highly efficient. The method is straightforward with hydrocarbon appendages but protection strategies are needed when reactive functionality is present. Functional groups present in the adduct must be fully compatible with the chemistry involved in the preparation and use of the phosphoramidite reagent and also with the conditions used in the final deprotection and cleavage from the solid support.

An adducted nucleoside approach has been developed for the preparation of oligonucleotides containing $\mathrm{N}$-acetyl-2-aminofluorene; the method allows the adduct to be placed in sequences that contain many guanines. ${ }^{11}$ Normal solid-phase phosphoramidite chemistry could not be used on account of the lability of the acetyl group to hot ammonia normally used for the final deprotection step. The problem was solved by using 9-fluorenylmethoxycarbonyl (FMOC) protection of the exocyclic amino groups; the FMOC group can be removed by the anion of 2nitrobenzaldoxime which does not displace the $\mathrm{N}$-acetyl group from the adduct (Scheme 4). 

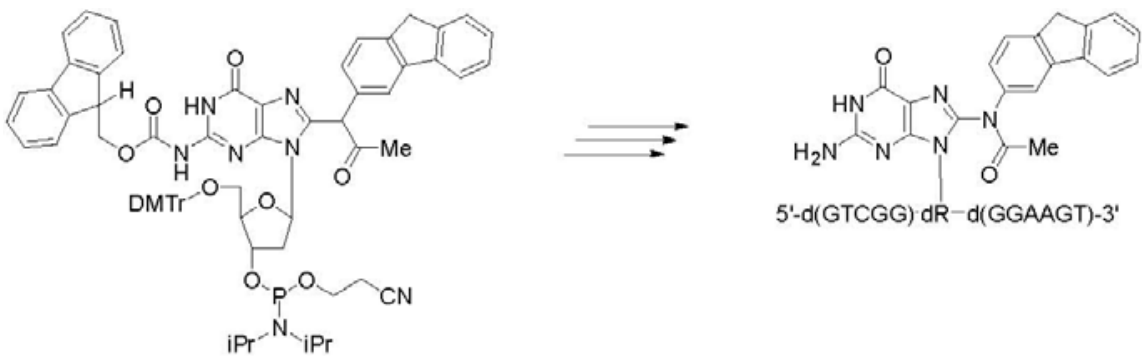

Scheme 4. Preparation of an oligodeoxynucleotide containing a guanine $\mathrm{C} 8$ adduct of $\mathrm{N}$-acetyl2-aminofluorene using the adducted nucleoside approach.

$O^{6}$-Alkyl derivatives of guanine are an interesting example of the use of the adducted nucleoside approach. The presence of adducts on the $\mathrm{O}^{6}$ position does not interfere with assembly of the oligonucleotide. Initial attempts to prepare oligonucleotides containing $O^{6}$ methylguanine were flawed by displacement of the methoxyl group by ammonia during the final deprotection step to produce a diamino purine residue. Deprotection with methanolic DBU circumvented this problem; even if displacement occurred, the substituent at $\mathrm{C} 6$ remains a methoxyl group. ${ }^{12,13}$ A subsequent enhancement is the use of a more labile protective group on the exocyclic amino groups; ammonia can then be used under mild enough conditions that the methoxyl group is stable. ${ }^{14,15}$ Recently the $\mathrm{O}^{6}$ adducted nucleoside methodology has been extended to the synthesis of an oligodeoxynucleotide having an $O^{6}$-(4-oxo-4-(3-pyridyl)butyl) adduct (Scheme 5). ${ }^{16}$ This adduct arises from 4-(methylnitrosamino)-1-(3-pyridyl)-1-butanone (NNK) which is one of most carcinogenic constituents of tobacco products and tobacco smoke. The keto group in the side chain represents an additional complication in the synthesis. The keto group had to be masked as a dithioketal prior to preparation of the nucleoside. After assembly of the oligodeoxynucleotide, the keto group was released by treatment with $N$-chlorosuccinimide.
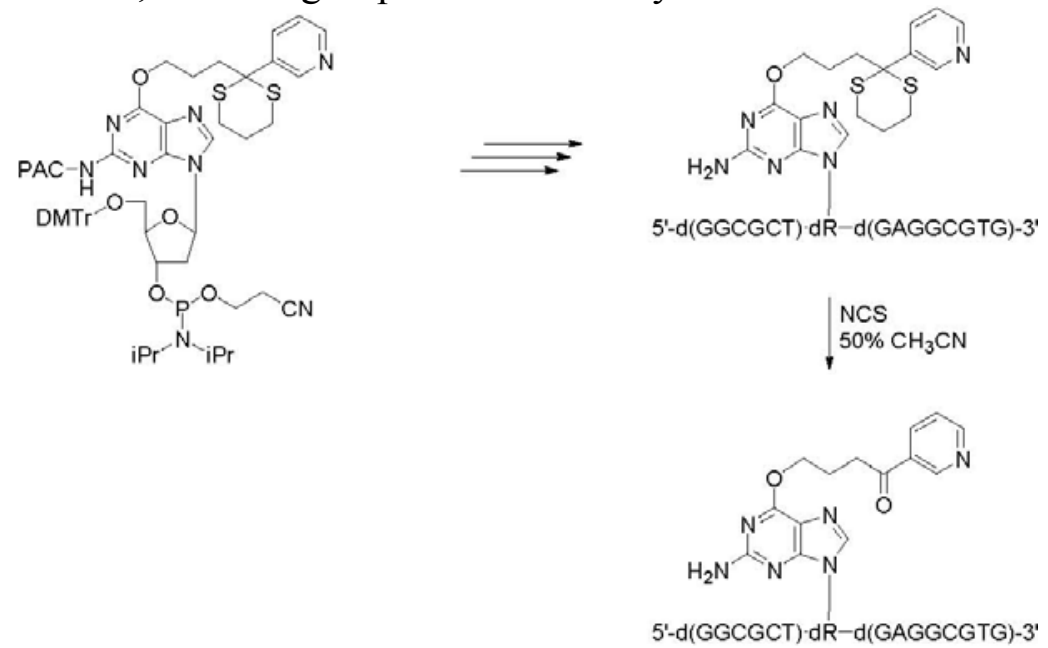

Scheme 5. Preparation of an oligonucleotide containing the 4-oxo-4-(3-pyridyl)butyl adduct at the $\mathrm{O}^{6}$ position of guanine using the adducted nucleoside approach. 
The preparation of oligonucleotides having structurally defined adducts of PAH diol epoxides is a synthetic challenge that has attracted considerable attention. The adducted nucleoside approach has been explored for cases where direct adduction is not suitable. One such case is the adenine adducts of the diol epoxides of benzo[a]pyrene because guanines react in preference to the adenines and even in the absence of guanine residues the yields for adenine adduction are too low to be synthetically useful. ${ }^{17,18}$ The adducted nucleosides were prepared with the PAH hydroxyl groups protected as benzoates (Scheme 6). Synthesis of the adducted nucleoside involved condensation of aminotribenzoates derived from the diol epoxides with 6fluoropurine 2'-deoxyriboside. The phosphoramidite reagents were then prepared in the usual fashion and used for assembly of oligonucleotides. The final deprotection step used hot ammonia, which also removed the benzoate groups. Extension of this strategy to adducts at the $\mathrm{N}^{2}$ position of guanine has been accomplished but is more difficult. ${ }^{19,20}$ The halogens at the 2 position of purine nucleosides are significantly less reactive than at the 6 , making it more difficult to prepare the benzoate-protected PAH-adducted nucleosides. Furthermore, bulky adducts on the exocyclic amino group of guanine impede the condensation step during oligonucleotide assembly, although it slow coupling can be offset by higher concentrations of the phosphoramidite reagent and/or longer reaction times. ${ }^{20,21} \mathrm{~A}$ third complication is that the overall yield based on utilization of the expensive PAH diol epoxides is low for the adducted nucleoside approach since they are introduced at an early stage in a synthetic scheme that requires many steps.

\section{Post-oligomerization}

The post-oligomerization approach is of value for placing adducts site-specifically on exocyclic amino groups of nucleosides. The general approach is to reverse the normal electrophilenucleophile functions of the mutagen and nucleoside such that an amine equivalent of the mutagen reacts with an electrophilic equivalent of the nucleoside in an aromatic nucleophilic substitution reaction. While this strategy has frequently been used for the preparation of adducted nucleosides as shown in the first step of Scheme 6, major advantages can arise from carrying the reaction out after assembly of an oligonucleotide. Specifically, it is no longer necessary to protect reactive functional groups on the appendage to avoid them interfering with assembly of the oligonucleotide. 


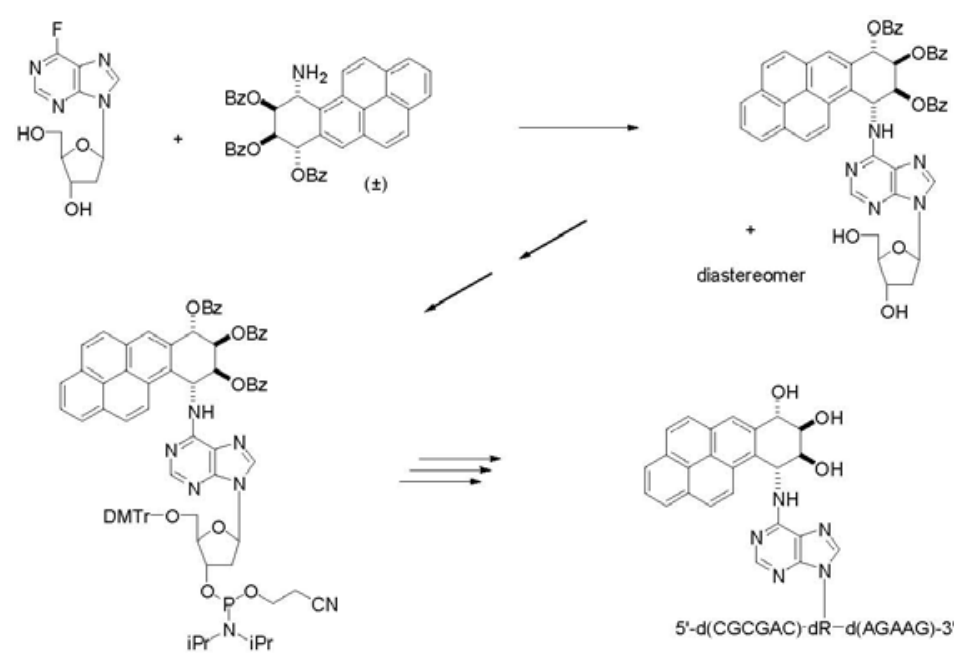

Scheme 6. Preparation of an oligonucleotide containing the $\mathrm{N}^{6}$ adenine trans adduct of the $(+)-$ anti dihydrodiol epoxide of benzo[ $a]$ pyrene using the adducted nucleoside approach.

Matteucci and Webb reported early examples of this strategy for preparation of oligonucleotides containing $N^{4}, N^{4}$-ethanodeoxycytidine and $N^{6}, N^{6}$-ethanodeoxy-adenosine, where the aziridine moiety would not have survived normal oligonucleotide deprotection conditions using extended treatment with hot ammonia. ${ }^{22-24}$ The method was later used by Benkovic $^{25}$ and Verdine. ${ }^{26}$

We have focused our attention on the use of the post-oligomerization strategy for placing mutagens site-specifically on the exocyclic amino groups of adenine and guanine. The initial goal of these studies was the preparation of oligonucleotides containing structurally defined adducts of the diol epoxides of benzo[ $[a]$ pyrene and other PAHs. The plan involved preparing oligonucleotides containing 2-haloinosine and 6-halopurine deoxynucleosides as synthetic equivalents of deoxyguanosine and deoxyadenosine and using the oligonucleotides in reactions with amino triols derived from the PAH diol epoxides. These condensations would provide not only the requisite specificity as to the site and stereochemistry of adduction but would also limit reaction of the mutagen synthon solely to the target nucleoside. This strategy would avoid the problems present in the direct adduction method of multiple target sites and incomplete specificity in the stereochemistry of epoxide opening. In addition, it would use unprotected amino triols, avoiding the cumbersome protection strategies required for the adducted nucleoside approach. It would offer economic advantages over adducted nucleosides since the amino triol would be introduced at the end of the synthesis rather than at the beginning. Furthermore, excess aminotriol, if required to promote the reaction, would be easily recovered, in contrast to the situation with the diol epoxides in the direct adduction method and the PAH-adducted phosphoramidite reagents in the adducted nucleoside method. This is an important feature since the diol epoxides from which the aminotriols are derived are laborious to prepare or expensive in cases where they can be purchased. 
In addressing adduction of deoxyadenosine, preliminary studies of the reaction of the trans aminotriol derived from the $( \pm)$ anti diol epoxide of benzo[a]pyrene with 6-halopurine deoxyribosides revealed that the chloronucleoside was insufficiently reactive. Satisfactory reaction rates were observed with simple, unhindered nucleophiles, but the reaction with the benzo[a]pyrene aminotriol was too slow to be useful in oligonucleotides. ${ }^{27}$ 6-Fluoropurine deoxyriboside showed enhanced reactivity giving satisfactory yields of the $\mathrm{N}^{6} \mathrm{PAH}$ adduct under conditions compatible with the envisioned reactions of oligonucleotides.

On the basis of this result, the strategy was then applied to the preparation of an adducted oligonucleotide. ${ }^{28}$ No problems were encountered in the preparation of the phosphoramidite reagent from the fluoropurine nucleoside or in the use of the phosphoramidite reagent in the assembly of an 11-mer oligonucleotide (Scheme 7). However, the lability of the fluoropurine precluded use of nucleophilic reagents to deprotect and cleave the oligonucleotide from the solid support. Consequently, the condensation was carried out with racemic amino triol while the oligonucleotide was still attached to the support.

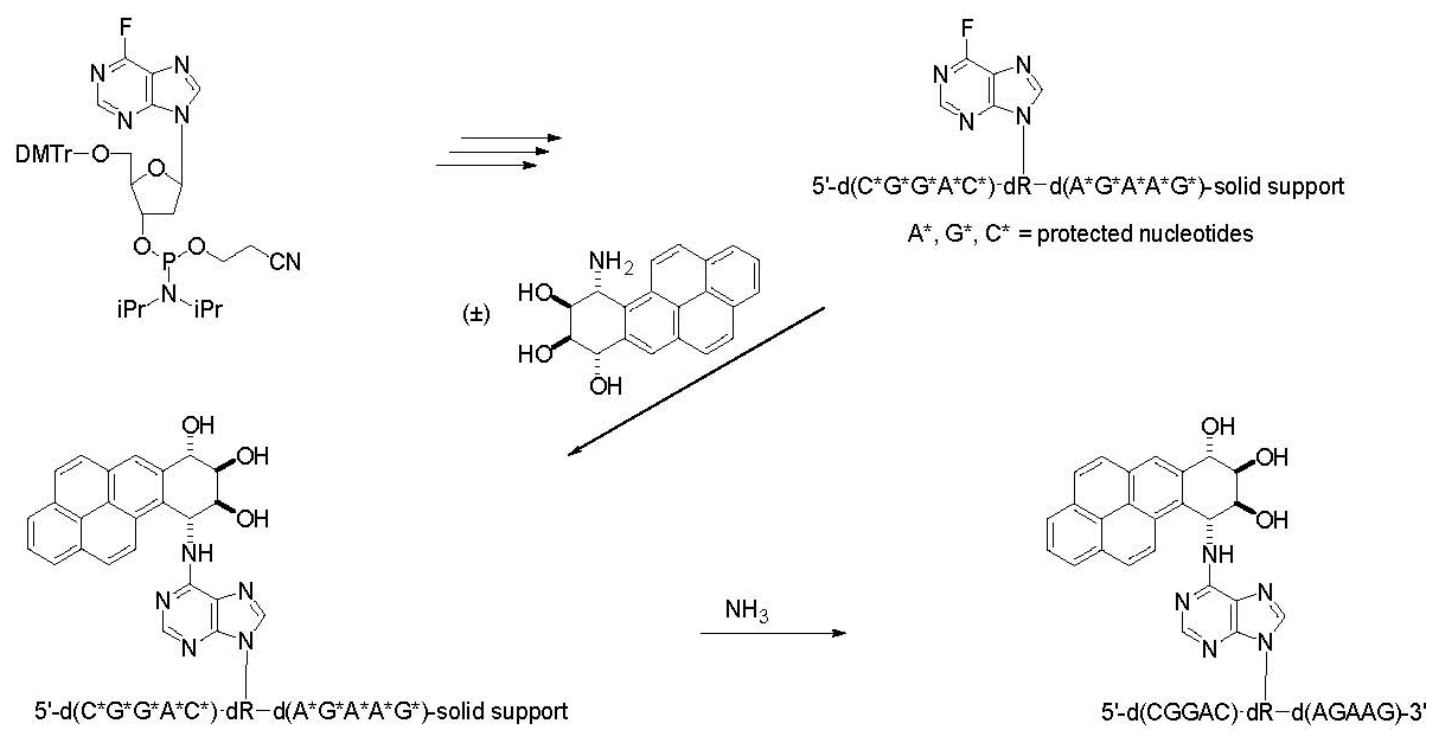

Scheme 7. Preparation of an oligonucleotide containing the $\mathrm{N}^{6}$ adenine trans adduct of the $(+)$ anti dihydrodiol epoxide of benzo[a]pyrene using the post-oligomerization approach.

After the reaction was complete, the beads were washed to remove excess aminotriol, treated with hot ammonia to achieve deprotection and the product then purified. It was found that the diastereomeric oligonucleotides resulting from using racemic aminotriol were readily resolved by reverse phase HPLC.

Problems were experienced with extension of this post-oligomerization strategy to the preparation of $\mathrm{N}^{2}$ adducts of guanine on account of the reduced reactivity of halogen at the 2 position on purines relative to the 6 position. Oligonucleotides containing 2-fluoroinosine deoxyriboside were found to react at a satisfactory rate with small, unhindered nucleophiles with 
which large excesses could be used. For example, reaction with phenylglycinol to give the $\mathrm{N}^{2}$ guanine adduct of styrene oxide worked well. ${ }^{29}$ However, reactivity with the aminotriol of benzo[a]pyrene was poor. Nevertheless, the post-oligomerization strategy has been used for preparation of oligonucleotides containing benzo[a]pyrene adducts on guanine (Scheme 8 ) ${ }^{20,30}$
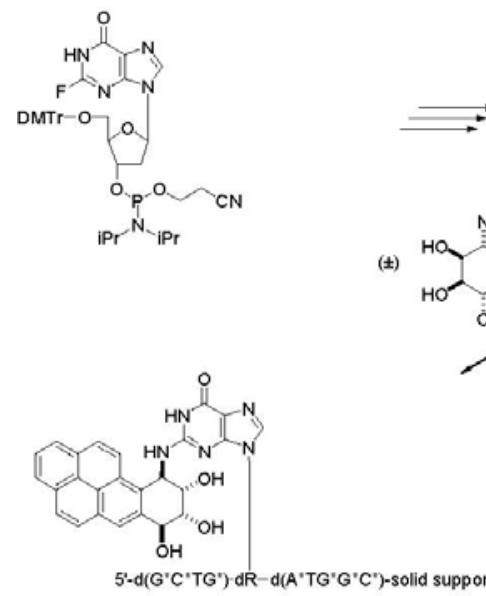

+ diastereomer
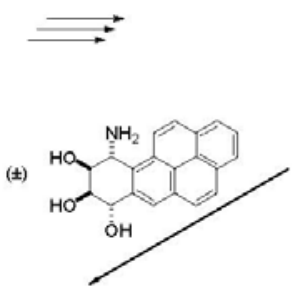



$A^{*}, G^{*}, C^{*}=$ protected nucleotides

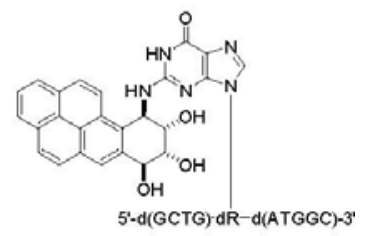

+ diastereomer

Scheme 8. Preparation of an oligonucleotide containing the $\mathrm{N}^{2}$ guanine trans adduct of the (+)anti dihydrodiol epoxide of benzo[ $a]$ pyrene using the post-oligomerization approach.

While searching for improved methodology for preparation of oligonucleotides having guanine $\mathrm{N}^{2}$ adducts, it was found that protection of the $\mathrm{O}^{6}$ position as an ether gave some improvement in rates of reaction of fluoroinosine oligonucleotides with nucleophiles. ${ }^{31}$ The trimethylsilyl ether is used advantageously for this purpose (Scheme 8). With fluoroinosine nucleoside the stability of the trimethylsilyl ether toward hydrolysis is good but the trimethylsilyl ethers of deoxyguanosine and $\mathrm{N}^{2}$ derivatives of deoxyguanosine are readily cleaved under mildly acidic conditions. Harvey and coworkers have used the 4-nitrophenylethyl ether similarly. ${ }^{19}$ The 4-nitrophenylethyl group is quite robust, requiring $0.5 \mathrm{M} \mathrm{DBU}$ in pyridine for cleavage.

The $O^{2}$-triflate of $O^{6}$-(4-nitrophenylethyl)deoxyxanthosine, offers substantial improvements over fluoride in reaction rates with nucleophiles. ${ }^{32}$ Good results have been obtained in the reaction of a 13-mer oligonucleotide containing this synthon with the aminotriol of benzo[a]pyrene (Scheme 9). ${ }^{33}$ The method has been used to prepare PAH-adducted oligonucleotides in quantities sufficient for NMR studies. 


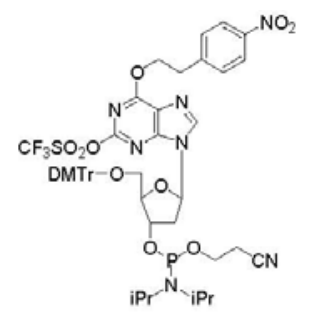

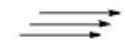
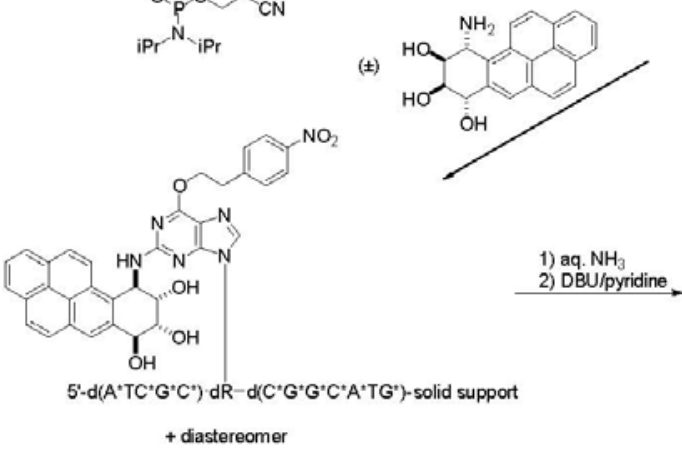

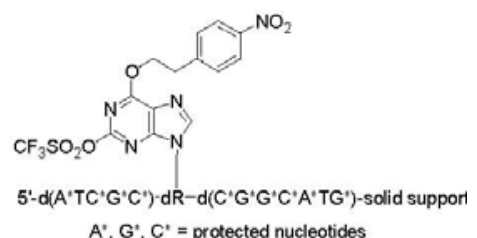

$A^{*}, G^{\prime}, C^{\prime}=$ protected nucleotides

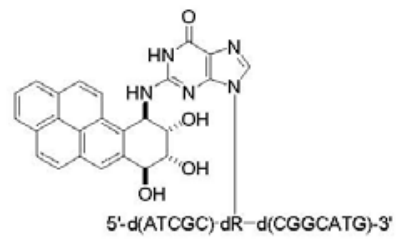

+ diastereomer

Scheme 9. Preparation of an oligonucleotide containing the $\mathrm{N}^{2}$ guanine trans adduct of the $(+)$ anti dihydrodiol epoxide of benzo[a]pyrene using the post-oligomerization approach.

While we were searching for better conditions for preparing PAH-adducted oligonucleotides, it was found that oligonucleotides containing 2-fluoro- $O^{6}$-(trimethylsilylethyl)inosine could be deprotected and removed from the solid support without loss of the fluoro or trimethylsilylethyl groups by treatment with $0.1 \mathrm{M} \mathrm{NaOH}\left(8 \mathrm{hr}, \mathrm{rt}\right.$ ) (Scheme 10a). ${ }^{31}$ The triflate derivative is too labile to survive this treatment. A similar approach has been successful for preparation of fully deprotected oligonucleotides containing 6-chloropurine but not 6-fluoropurine (Scheme 10b). ${ }^{34}$ These procedures offer the advantage that the oligonucleotides containing the guanine and adenine synthons can be purified prior to reaction with nucleophiles. Other advantages are that displacement reactions can be carried out in homogeneous solution rather than on the solid support and the progress of reactions can easily be monitored by HPLC or other analytical methods. The homogeneous methods are useful for reactions with unencumbered nucleophiles and they have been used to prepare oligonucleotides containing adducts of styrene oxide, the monoepoxide of butadiene and the epoxide of 1,2-dihydroxy-3-butene. ${ }^{34,35}$ HPLC purification is carried out first at the halopurine stage and again with the adducted oligonucleotide, making it easier to obtain the high purity needed for mutagenesis studies.

The homogeneous methods have been used to advantage for synthesis of DNA containing interchain crosslinks. ${ }^{34,36-38}$ Examples include dehydroretronecine and trimethylene crosslinks between guanines in a self-complementary sequence, a trimethylene crosslink between guanines in a non-self-complementary sequence, and a tetramethylene crosslink between adenines, also in a non-self-complementary sequence (Scheme 11). 


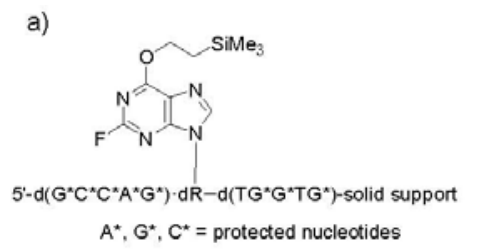

b)

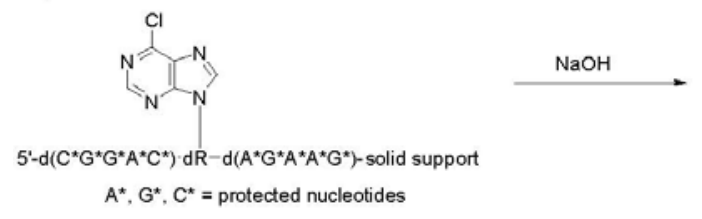

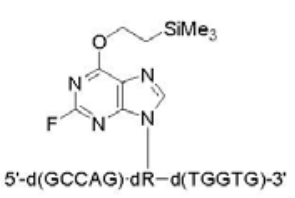

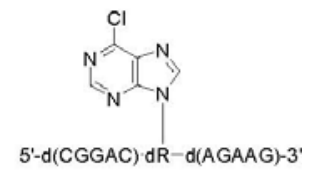

Scheme 10. Preparation of deprotected oligonucleotides containing halopurine equivalents of guanine and adenine.

Crosslinking reactions carried out on solid supports would be limited to crosslinks between identical chains and might be unsatisfactory even for symmetrical crosslinks. Homogeneous reaction conditions have also been used for synthesis of intrastrand crosslinks between adjacent guanine residues; four-, three-, and two-carbon tethers have been produced in this manner. ${ }^{39}$
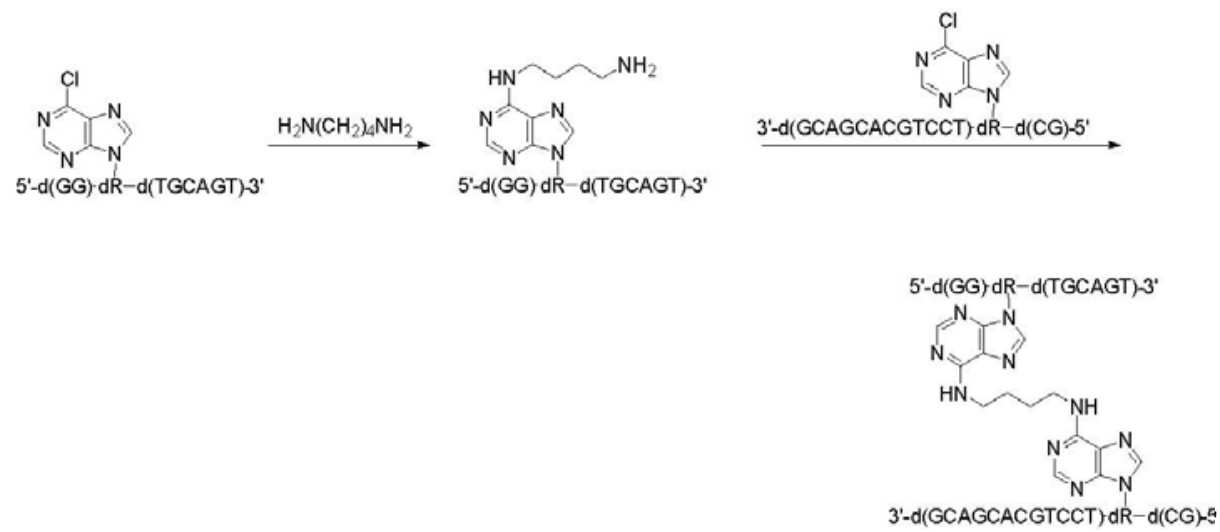

Scheme 11. Preparation of a 9-mer:15-mer duplex containing a 4-carbon tether between adenines.

A recent application of homogeneous reaction conditions has been the synthesis of oligonucleotides containing adducts of acrolein and the epoxide of vinyl chloride. ${ }^{40}$ These biselectrophiles form $1, N^{2}$-hydroxypropano and hydroxyethano cyclic adducts with guanine. The post-oligomerization strategy would require use of $\beta$-amino-propionaldehyde and $\alpha$ aminoacetaldehyde, respectively, to form these adducts. However, these aminoaldehydes are unstable, precluding their use in this manner. To circumvent this problem, $\omega$-amino-1,2-diols were used as surrogates for the aminoaldehydes; the diols were cleaved with $\mathrm{NaIO}_{4}$ as the final step in the synthesis. 

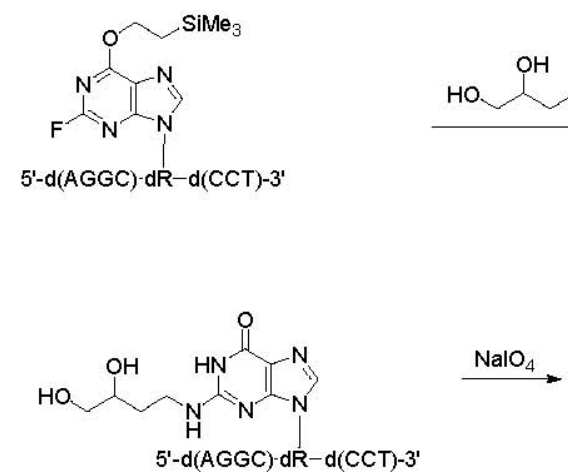
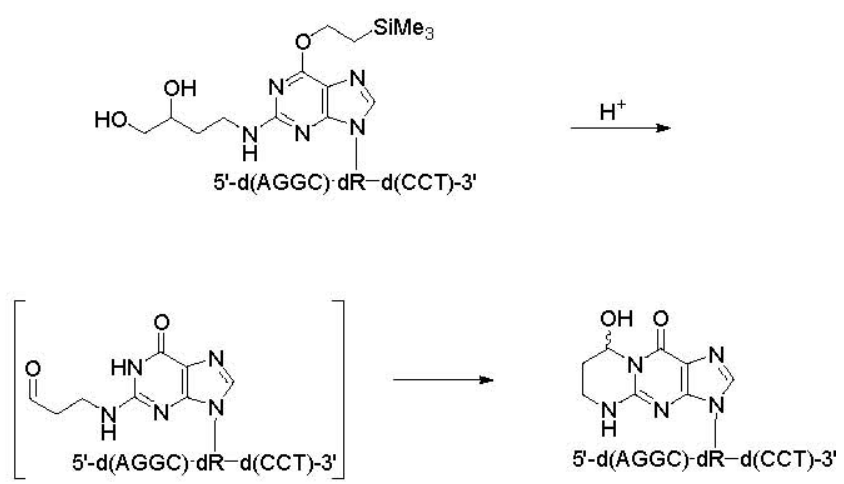

Scheme 12. Preparation of an 8-mer containing an acrolein adduct on guanine.

\section{Conclusions}

Three methods are presented for preparing DNA containing structurally defined adducts: direct adduction, adducted nucleoside and post-oligomerization. This review focuses on the adducts of mutagens, but the methodology presented is equally applicable for the preparation of other types of adducts. Each of the methods has its strengths and weaknesses. Direct adduction is attractive when the oligonucleotide contains only a single target site and the efficiency of adduction is good. The adducted nucleoside approach is highly effective with unfunctionalized adducts, if they do not sterically hinder oligonucleotide assembly. The post-oligomerization approach has wide applicability for the preparation of adducts on the exocyclic amino groups of the bases and is particularly useful for adducts containing functional groups that would interfere with oligonucleotide synthesis and for the preparation of interchain and intrachain crosslinks.

\section{Acknowledgements}

We gratefully acknowledge research support from the National Institute for Environmental Health Sciences (ES00267, ES07781 and ES05355).

\section{References and Notes}

1. Baertschi, S. W.; Raney, K. D.; Stone, M. P.; Harris, T. M. J. Am. Chem. Soc. 1988, 110, 7929.

2. Johnson, W. W.; Harris, T. M.; Guengerich, F. P. J. Am. Chem. Soc. 1996, 118, 8213.

3. Gopalakrishnan, S.; Stone, M. P.; Harris, T. M. J. Am. Chem. Soc. 1989, 111, 7232.

4. Jones, W. R.; Stone, M. P. Nucleic Acids Res. 1998, 26, 1070. 
5. Iyer, R. S.; Coles, B. F.; Raney, K. D.; Thier, R.; Guengerich, F. P.; Harris, T. M. J. Am. Chem. Soc. 1994, 116, 1603.

6. Kobertz, W.; Wang, D.; Wogan, G. N.; Essigmann, J. M. Proc. Natl. Acad. Sci. U.S.A. 1997, 94, 9579.

7. Cosman, M.; Ibanez, V.; Geacintov, N. E.; Harvey, R. G. Carcinogenesis (London) 1990, 11, 1667.

8. Laryea, A; Cosman, M.; Lin, J.-M.; Liu, T.; Agarwal, R.; Smirnov, S.; Amin, S.; Harvey, R. G.; Dipple, A.; Geacintov, N. E. Chem. Res. Toxicol. 1995, 8, 444.

9. Koeh1, P.; Burnouf, D.; Fuchs, R. P. P. J. Mol. Biol. 1989, 207, 355.

10. Ezaz-Nikpay, K.; Verdine, G. L. J. Am. Chem. Soc. 1992, 114, 6562.

11. Zhou, Y.; Romano, L. J. Biochemistry 1993, 32, 14043.

12. Kuzmich, S.; Markey, L. A.; Jones, R. A. Nucleic Acids Res. 1983, 11, 3393.

13. Borowy-Borowski, H.; Chambers, R. W. Biochemistry 1987, 26, 2465.

14. Schulhof, J. C.; Molko, D.; Teoule, R. Nucleic Acids Res. 1987, $15,397$.

15. Li, B. F. L; Swann, P.F. Biochemistry 1989, 28, 5779.

16. Wang, L.; Spratt, T. E.; Pegg, A. E.; Peterson, L. A. Chem. Res. Toxicol. 1999, 12, 127.

17. Lakshman, M. K.; Sayer, J. M.; Jerina, D. M. J. Am. Chem. Soc. 1991, 113, 6589.

18. Chaturvedi, S.; Lakshman, M. K. Carcinogenesis 1996, 17, 2747.

19. Lee, H.; Hinz, M.; Stezowski, J. J.; Harvey, R. G. Tetrahedron Lett. 1990, 31, 6773.

20. Custer, L.; Zajc, B.; Sayer, J. M.; Cullinane, C.; Phillips, D. R.; Cheh, A. M.; Jerina, D. M.; Bohr, V. A.; Mazur, S.J. Biochemistry 1999, 38, 569.

21. Casale, R.; McLaughlin, L. W. J. Am. Chem. Soc. 1990, 112, 5264.

22. Webb, T. R.; Matteucci, M. D. Nucleic Acids Res. 1986, 14, 7661.

23. Webb, T. R.; Matteucci, M. D. J. Am. Chem. Soc. 1986, 108, 2764.

24. Matteucci, M. D.; Webb, T. R. Tetrahedron Lett. 1987, 28, 2469.

25. Cowart, M.; Gibson, K. J.; Allen, D. J.; Benkovic, S. J. Biochemistry 1989, 28, 1975.

26. MacMillan, A. M.; Verdine, G. L. Tetrahedron 1991, 47, 2603.

27. Kim, S. J.; Harris, C. M.; Harris, Jung, K.-Y.; Koreeda, M.; Harris, T. M. Tetrahedron Lett. 1991, 32, 6073.

28. Kim, S.J.; Stone, M. P.; Harris, C. M.; Harris, T. M. J. Am. Chem. Soc. 1992, 114, 5480.

29. Harris, C.M.; Zhou, L.; Strand, E. A.; Harris, T. M. J. Am. Chem. Soc. 1991, 113, 4328.

30. Page, J. E.; Zajc, B.; Oh-hara, T.; Lakshman, M. K.; Sayer, J. M.; Jerina, D. M.; Dipple, A. Biochemistry 1998, 37, 9127.

31. DeCorte, B. L.; Tsarouhtsis, D.; Kuchimanchi, S.; Cooper, M. D.; Horton, P.; Harris, C. M.; Harris, T. M. Chem. Res. Toxicol. 1996, 9, 630.

32. Steinbrecher, T.; Wameling, C.; Oesch, F.; Seidel, A. Angew. Chem., Int. Ed. 1993, 32, 404.

33. Cooper, M. D.; Hodge, R. P.; Tamura, P. J.; Wilkinson, A. S.; Harris, C. M.; Harris, T. M. Tetrahedron Lett. 2000, 41, 3555.

34. Kim, H.-Y.; Nechev, L.; Zhou, L.; Tamura, P.; Harris, C. M.; Harris, T. M. Tetrahedron Lett. 1998, 39, 6803. 
35. Nechev, L. V.; Zhang, M.; Tsarouhtsis, D.; Tamura, P. J.; Wilkinson, A.S.; Harris, C. M.; Harris, T. M. Chem. Res. Toxicol. 2001, 14, 379.

36. Tsarouhtsis, D.; Kuchimanchi, S.; DeCorte, B.L.; Harris, C. M.; Harris, T. M. J. Am. Chem. Soc. 1995, 117, 11013.

37. Dooley, P. A.; Tsarouhtsis, D.; Nechev, L. V.; Korbel, G. A.; Shearer, J.; Zegar, I. S.; Harris, C. M.; Stone, M. P.; Harris, T. M. J. Am. Chem. Soc. 2001, 123, 1730.

38. Mu, D.; Bessho, T.; Chen, D. J.; Harris, T. M.; Nechev, L. V.; Hearst, J. E.; Sancar, A. Molec. Cell. Biol. 2000, 20, 2446.

39. Carmical, J. R.; Zou, Y.; Van Houten, B.; Kowalczyk, A.; Nechev, L. V.; Harris, C. M.; Harris, T. M.; Lloyd, R. S. J. Biol. Chem. 2000, 275, 19482.

40. Nechev, L. V.; Harris, C. M.; Harris, T. M. Chem. Res. Toxicol. 2000, 13, 421. 